\title{
Development of a positioning aid to reduce postural variability and errors in 3D whole body scan measurements
}

\section{Author names and affiliations}

\author{
Frank Schwarz-Müller ${ }^{\text {a, b * }}, \quad$ F.Schwarz-Muller@lboro.ac.uk \\ Dr Russell Marshall a , $\quad$ R.Marshall@lboro.ac.uk \\ Dr Steve Summerskill a , $\quad$ S.J.Summerskill2@lboro.ac.uk \\ ${ }^{a}$ Loughborough Design School, Loughborough University, Leicestershire LE11 3TU, UK \\ ${ }^{\mathrm{b}}$ Federal Ministry of Defence, Fontainengraben 150, 53123 Bonn, Germany
}

\begin{abstract}
Three-dimensional (3D) body scanners have the potential to evaluate changes to the human form through different clothing configurations, the use of protective equipment, or the effects of medical interventions. To achieve this, scans of an individual need to be superimposed for each experimental condition. The literature highlights that one of the limiting factors is postural variability. This paper describes a newly developed 'positioning aid' that stabilises the posture during the scanning process and is invisible on scans. The results of a study evaluating the efficacy of the positioning aid showed that it reduces postural variability for all body parts in lateral and longitudinal directions. A reference test with a rigid mannequin indicated that the 'technical' variability due to the scanner hardware and software significantly contributes to the residual variability. Furthermore, the study showed that the newly developed positioning aid overall increased the precision of the software-assisted extraction of body dimensions.
\end{abstract}

\section{Key words}

Body scanner; posture; precision; positioning aid

\footnotetext{
* Corresponding author, F.Schwarz-Muller@lboro.ac.uk
} 


\section{Introduction}

\subsection{Factors influencing precision of data captured with 3D body scanners}

The introduction of 3D body scanners has revolutionised the capture of anthropometric data since they allow automatic, rapid and contact free data collection (Daanen and van de Water, 1998; Robinette and Daanen, 2006). These significant advantages compared to the traditional manual anthropometric measurements have resulted in the use of 3D body scanning technology in numerous anthropometric surveys worldwide ( $\mathrm{Lu}$ et al., 2010; Treleaven, 2004).

As a result, the number of anthropometric databases based on data obtained with 3D body scanners is constantly growing. It is therefore of particular importance that these data exhibit a high degree of accuracy and precision. ISO 20685, the standard for 3D scanning methodologies for internationally compatible anthropometric databases, defines accuracy as the extent to which a measurement extracted from a 3D scan approximates the reference value (EN ISO, 2010). The reference value of body dimensions is determined by a skilled researcher utilising traditional instruments such as callipers, stadiometers, anthropometers, sitting height tables and measuring tapes. The precision of scan derived measurements, also referred to as repeatability, is defined as the difference between multiple measurements with the same 3D scanning system (Lu and Wang, 2010).

Accuracy and precision of the data ascertained with 3D body scanners are influenced by the factors listed in Figure 1 and are broadly split into two main categories: Technical Variability and Human Variability (Kouchi and Mochimaru, 2011, 2008; Mckinnon and Istook, 2002):

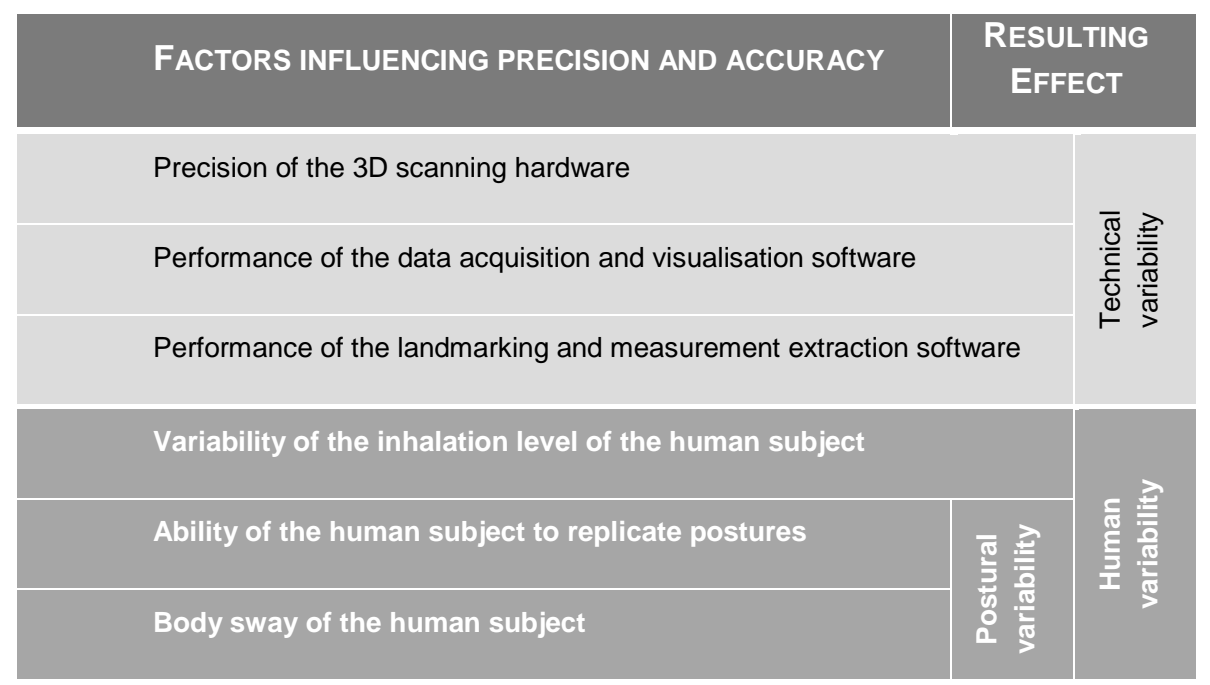

Figure 1: Factors affecting precision and accuracy in 3D body scanning

Previous studies have shown that body dimensions extracted from 3D body scans regularly fail to satisfy the accuracy requirements laid down in ISO 20685 for the use in anthropometric databases (Han et al., 2010; Lu and Wang, 2010; Mckinnon and Istook, 2002). Thus, those of the aforementioned factors need to be identified, which potentially inhibit achieving the desired level of accuracy and precision. 
Most manufacturers offer their 3D body scanning systems as a package comprising of the scanner, controllers to operate the scanner as well as IT for data processing and storage. In the majority of cases they also provide a proprietary software package dedicated to data acquisition, anatomical landmark detection and automatic measurement of body dimensions (D’Apuzzo, 2007). Thus, it can be inferred that the factors influencing the technical variability (Figure 1) are system inherent. The only remaining option for users to reduce the technical variability is to make sure that their scanning system is as up-to-date as possible and calibrated appropriately.

ISO 20685 stipulates that "for all postures, quiet respiration (normal breathing) should be adopted". This is in line with the findings of the study conducted by Mckinnon and Istook (Mckinnon and Istook, 2002), who scanned subjects holding their breath at different inhalation levels and when breathing normally. They found that humans are unable to reliably replicate a certain respiration level and that continuing breathing normally during the scanning process compromises the data integrity least.

It is common practise that the subjects are verbally instructed by the scanner operator how to adopt the scanning posture. Since they are either standing or sitting unrestrained on the scanner platform, variations in the posture due to body sway and the user's inability to replicate postures are inevitable. Thus, in a number of studies postural variability was found to compromise the integrity of the scan derived anthropometric data considerably (Han et al., 2010; Lu and Wang, 2010; Mckinnon and Istook, 2002).

Therefore, to achieve greater precision in body scan data capture, the main focus should be to address the replication of a consistent posture.

\subsection{Reduction of human variability by means of a positioning aid}

Although a number of researchers called for measures to stabilise the human posture during the scanning process (Lu and Wang, 2010; Mckinnon and Istook, 2002; Tomkinson and Shaw, 2013), so far only a few attempts have been made to counter body sway and poor posture replication. Exceptions are handles to stabilise the arm posture that can be found in body scanners manufactured by $\mathrm{TC}^{2}$ and Size Stream or rudimentary fixation elements used in studies conducted by Reed and Guitierrez (Guitierrez, C. M. and Gallagher, 2008; Reed, 2012). A reason for a lack of research in this field might be the fact that mechanical positioning aids potentially compromise the scan data by obscuring relevant body parts. As a result, the software-assisted extraction of body dimensions does not work reliably anymore.

In their article "The Evaluation of Scan-Derived Anthropometric Measurements" Lu and Wang (2010) reported that they used a rigid mannequin to entirely eliminate the effects of the human variability. In contrast to the scan derived measurements obtained from human subjects, those ascertained from the mannequin met the accuracy and precision requirements of ISO 20685 for the use in anthropometric databases. This is a strong indication that scan derived measurements of human subjects can achieve the desired level of precision provided that the human variability can be limited efficiently. It can therefore be inferred that the reduction of postural variability by means of a positioning aid presents an opportunity to increase the precision of scan derived measurements. 
An additional driver for the implementation of a successful positioning aid is to address emerging applications of 3D body scanning technology. These emerging applications require the precise superimposition of 3D images of the same subject obtained at different occasions or in diverse configurations. This would for instance allow analysing the effects of medical treatments, diets or workout by comparing aligned scans of the subject before and after the intervention. Another application is "clothed anthropometry" (Hsiao et al., 2014; Stewart et al., 2016). Although it seems to be obvious that wearing personal protective equipment (PPE) leads to an increased space claim there is no standardized methodology in place to quantify it by means of 3D body scanning (Jones et al., 2015). The comparison of subjects scanned in the standard scanning attire (tight underwear) and fully encumbered by PPE would allow the space claim differences to be established. Determining the linear, circumferential or volumetric difference between different clothing configurations obtained in the same scanning posture necessitates the precise superimposition of scans (Figure 2).
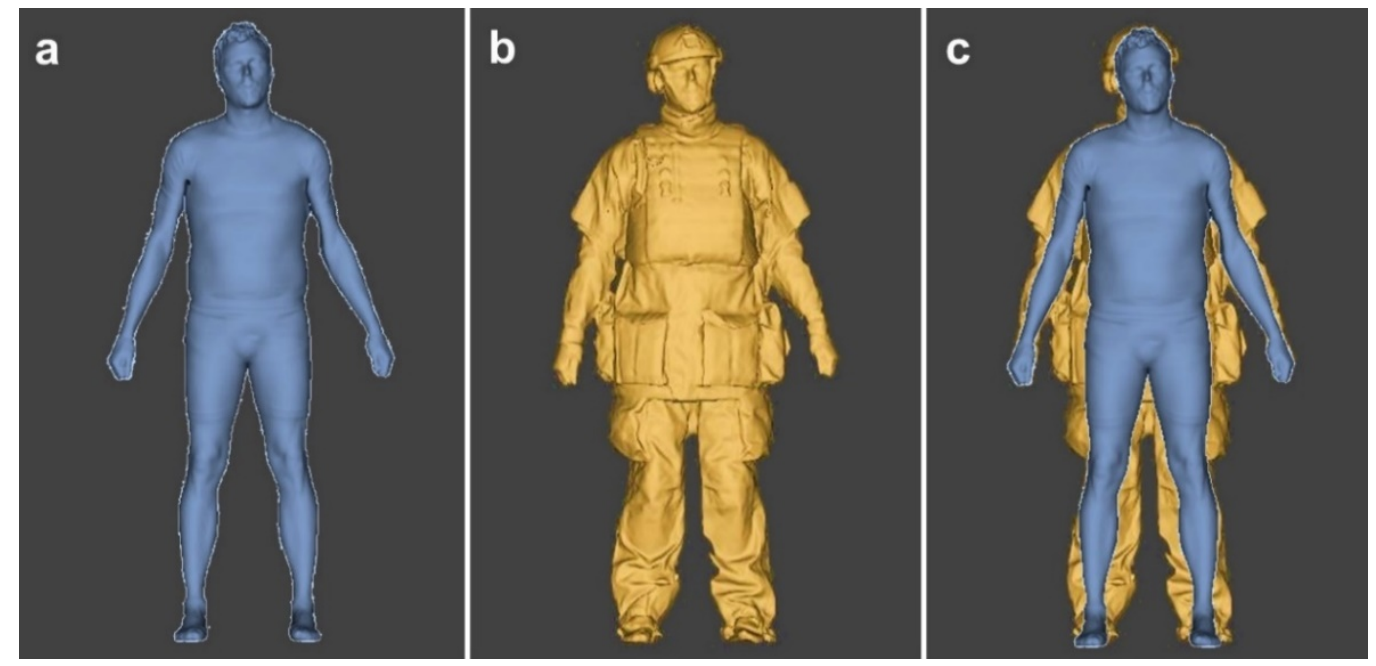

Figure 2: Superimposition of scans (c) to determine the difference between clothing configurations (a, b)

A further potential application for a positioning aid is the analysis of 3D data where the harmonisation of the postures across a sample of different individuals is desirable (e.g. principle components analysis).

The following sections describe the development of a positioning aid, the design of which has been systematically optimised to restrain body movement without compromising scan quality. Subsequently, it presents the results of a study conducted to analyse the effectiveness of the newly developed positioning aid in reducing postural variability, i.e. body sway and poor replication of the standard scanning posture. 


\section{Methods}

\subsection{Development of a positioning aid}

\subsubsection{Selection of support elements}

The literature review has shown that only a few studies have been conducted to date to address positioning, replication of postures and reduction of movement artefacts. In the article "Reducing movement artefacts in whole body scanning" (Daanen et al., 1997), it was reported that by reducing the head movement of an unrestrained subject the longitudinal sway of the body during a scan can be reduced significantly. Furthermore, the average lateral body sway was found to be negligible compared to the scanner resolution (2-4 mm). Finally, a comparison of two subsequent scans revealed that movement artefacts were also caused by differences in the hip abduction. Significant deviations of hip related measurements derived from multiple scans were also reported by Mckinnon and Istook (2002).

Since lateral body sway was found to be negligible it was not taken into consideration. However, it was unquestionable that the positioning aid needs to provide a feature avoiding the variation of the hip abduction during multiple scans.

Posture variation of the extremities make it particularly difficult to properly align scans. The manufacturer of the scanner (VITRONIC) used for the study stipulates that in the standard standing scanning posture arms have to be stretched out with a distance of $20 \mathrm{~cm}$ from the thighs in the centre of the body with hands in a fist.

The foot span recommended by the scanner manufacturer minimises occlusions and consequently improves the scan quality.

It can therefore be inferred that a positioning aid needs to incorporate fixtures for head, arms and feet as well as a support element for the hip to avoid the most eminent effects of postural variability.

\subsubsection{Evolution of the Positioning Aid}

An essential requirement for the positioning aid is that it reliably reduces the postural variability during the scanning process. Furthermore, it is crucial that it does not occlude relevant parts of the body or cause artefacts compromising the scan quality. To achieve the latter, the system inherent limitations of the predominant body scanning technologies play a decisive role. The sensors of both, laser and white light scanners can only pick up light that is reflected from the scanned object. Taking this into account, the first generation of the positioning aid incorporated the following design features (Figure 3 and Figure 4):

Head fixture - A chin rest in combination with a forehead support, as known from medical applications, was integrated in a U-shaped steel frame as head fixture.

Arm fixture - Handles mounted to telescopic steel tubes and positioned on either side of the body were utilised as combined arm and hand fixture. 
Hip support - A translucent Plexiglas tube was placed horizontally in front of the body, allowing subjects to move the hip forward until it slightly touches the tube to avoid variations in hip abduction.

Foot fixtures - Two shoe-measuring-gauges formed like a footprint and incorporating heel cups, into which the subject is expected to firmly press their heels, were fixed at a distance of $35 \mathrm{~cm}$ apart as foot fixtures. This distance is stipulated by the scanner manufacturer for the standard standing posture (identical with distance of the footprints on the scanner platform).

Except the Plexiglas tube of the hip support all elements were coated with matt black paint to minimise light reflection (Figure 3).
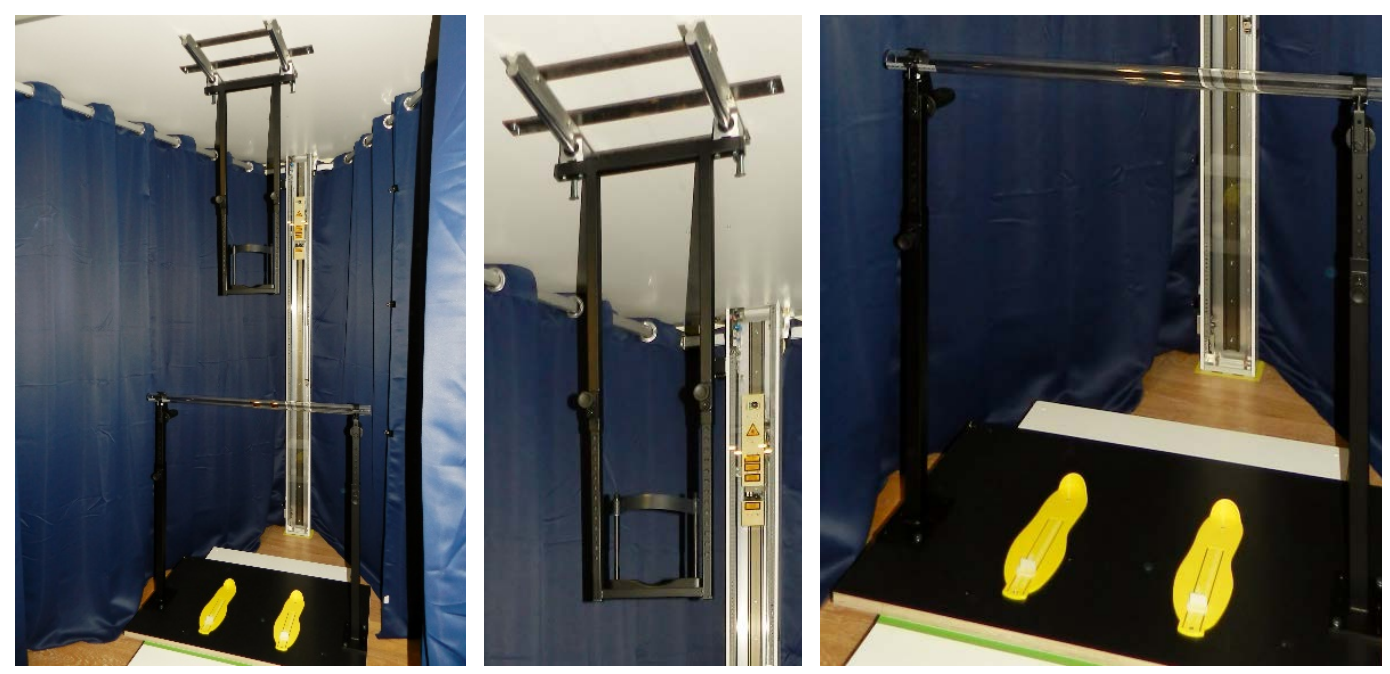

Figure 3: Fixation elements of the positioning aid $-1^{\text {st }}$ Generation 
The adjustment ranges of the fixation elements are depicted in Figure 4.
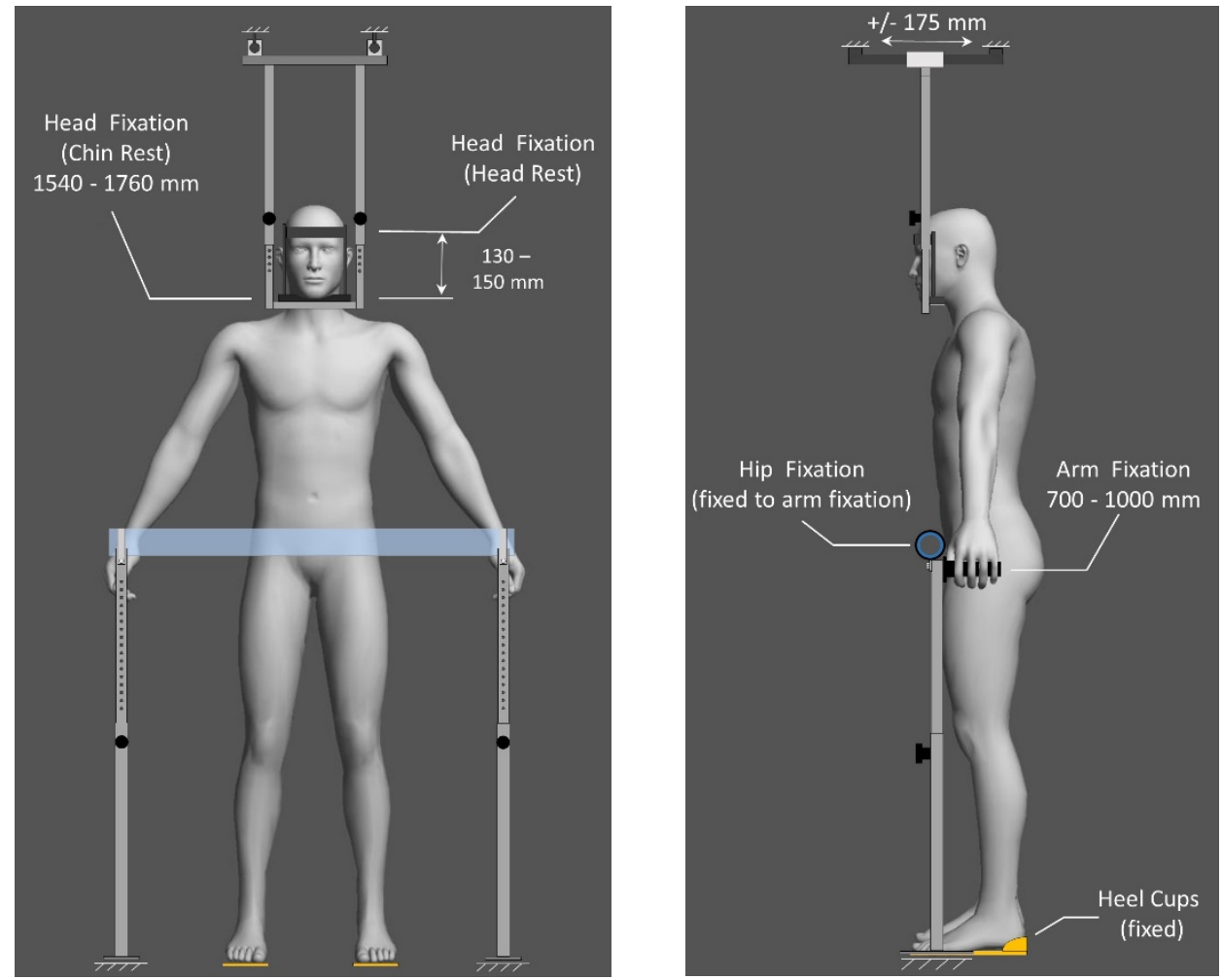

Figure 4: Adjustability of the positioning aid $-1^{\text {st }}$ Generation

A pilot study with three participants was carried out to assess the usability of the positioning aid and to analyse to what extent it compromises the scan quality. The tests were conducted with a "VITUS smart LC" scanner (VITRONIC) in combination with the "Anthroscan" software (Human Solutions). The scans of a human subject restrained by the positioning aid showed that the positioning aid affects the integrity of the scan data adversely (Figure 5).
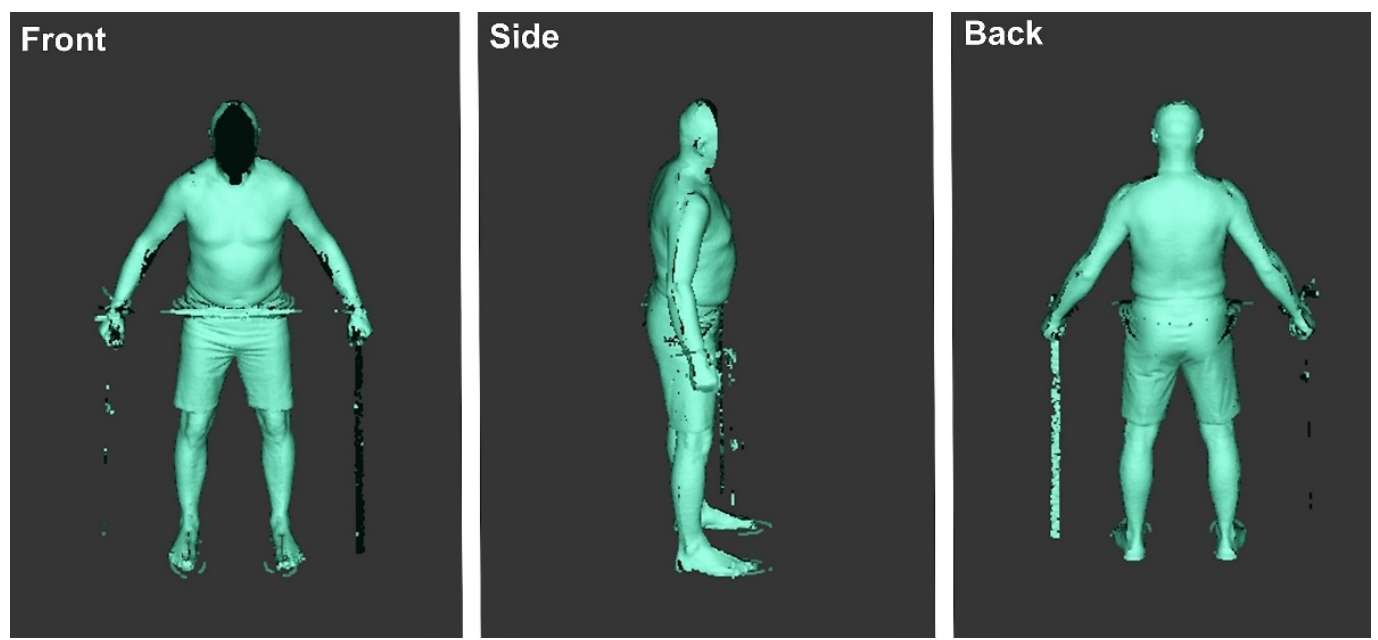

Figure 5: VITUS smart LC scan of a human subject restrained by positioning aid $-1^{\text {st }}$ Generation 
Although coated with matt black paint, parts of the telescopic tubes of the handles remained visible. Furthermore, the Plexiglas tube caused artefacts in the hip area, which are likely to result from light refraction. As far as the head fixture was concerned the matt black coating met the expectation and made the U-frame, including the chinrest and forehead support, invisible. However, the face of the subject was not displayed on the scan either. On the scans taken during pre-tests with the unpainted head fixture both, the U-frame with chinrest and forehead support as well as the head of the subject remained visible. The conclusion is that the black coated U-frame surrounding the head of the subject results in a point cloud, which the software is unable to separate into different objects (in contrast to the unpainted head fixture), nor can it be identified as part of the body. Thus, the point cloud from the tip of the nose to the rearward edge of the U-frame is not displayed on the scan. If relevant parts of the body are either occluded by elements of the positioning aid or even not displayed, a reliable software-supported extraction of body dimensions is impossible.

Hence, a fundamental redesign of the positioning aid was required. The design efforts for the " $2{ }^{\text {nd }}$ generation" positioning aid focused on replacing all black coated design elements by Plexiglas solutions. Since Plexiglas located too closely to the body potentially causes artefacts on the scan due to refraction considerable effort was expended in finding the optimal distance to the body without limiting the fixation properties.

The design of the $2^{\text {nd }}$ generation positioning aid incorporated the following features (Figure 6):

Head fixture - A mouthpiece incorporating a stop collar the subject is supposed to bite down upon was fixed to one end of a $400 \mathrm{~mm}$ long solid Plexiglas rod with a diameter of $8 \mathrm{~mm}$. The opposite end of the rod was inserted in holes drilled through the bottom end of a vertical $40 \mathrm{~mm}$ Plexiglas tube.

Arm fixture - To ensure an adequate stiffness translucent Plexiglas tubes with a diameter of $56 \mathrm{~mm}$ and a material thickness of $3 \mathrm{~mm}$ were selected to replace the telescopic steel tubes found in the previous version. Centred holes were drilled into the upper end of the tubes in longitudinal direction. Plexiglas tubes with a diameter of $14 \mathrm{~mm}$ (150 mm long) inserted in these holes from the front served as handles.

Hip support - A steel wire with a diameter of $2 \mathrm{~mm}$ fixed on either side to telescopic steel tubes positioned outside the scan volume was provided as hip support. Moving the hip forward until it slightly touches the taut steel wire avoids variations in hip abduction during the scanning process. Given the resolution of a 3D body scanner of 2-4 mm the steel wire remains invisible on the scan.

Foot fixtures - The foot fixtures remained unchanged. 

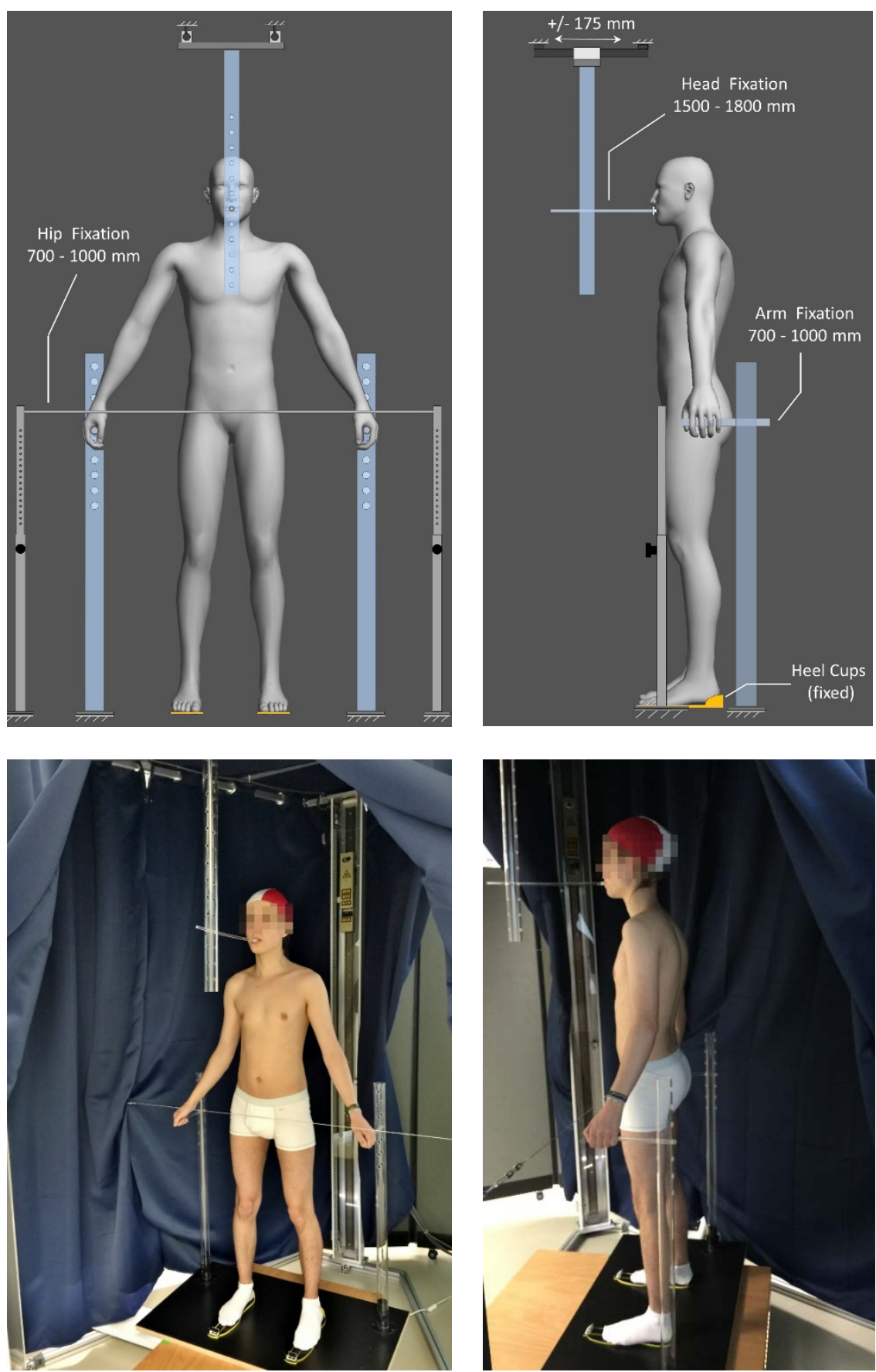

Figure 6: Fixation elements and adjustability of the positioning aid $-2^{\text {nd }}$ Generation

The design measures taken to minimise the impact of the positioning aid on data integrity and scan quality led to the desired result. In its final configuration neither the fixation elements themselves nor artefacts due to light refraction of the Plexiglas components were visible on the scans (Figure 7). 

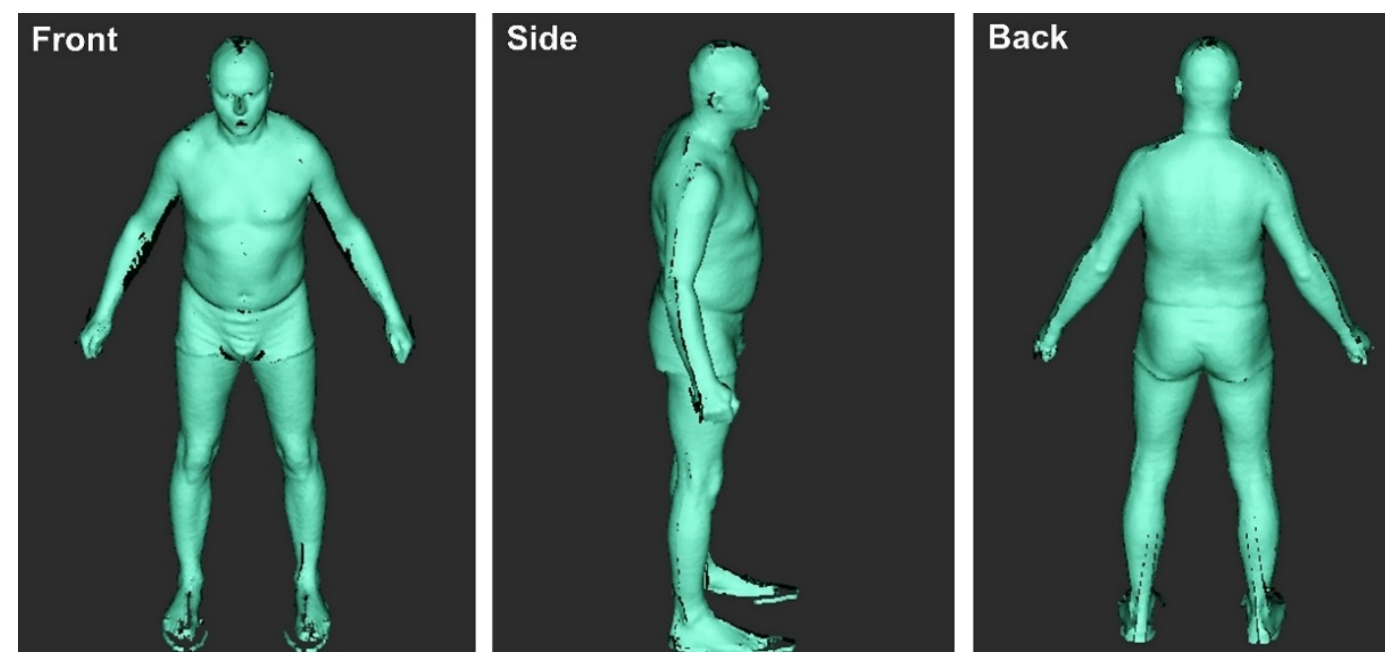

Figure 7: Scan of a human subject restrained by positioning aid $-2^{\text {nd }}$ Generation

The analysis of the scans showed that merely the contours of the foot fixtures and a small part of the mouthpiece made of white plastic remained visible on the scans produced with the VITUS smart LC laser scanner (Figure 7).

Based on these findings the design goals for the positioning aid were considered to be met and its design judged to be mature for testing.

\subsection{Methodology for evaluating the effectiveness of the positioning aid}

\subsubsection{Design of the experiment}

After the design of the positioning aid had been systematically optimised to restrain body movement without compromising the scan quality a study was conducted to analyse its effectiveness in reducing body sway and posture variability.

15 human subjects were recruited to participate in the study (4 females and 11 males), ranging in age from 19 to 54 (mean $=38.4$, standard deviation $=11.58)$ and in body height from $1599 \mathrm{~mm}$ to $1941 \mathrm{~mm}$ (mean = $1762 \mathrm{~mm}$, standard deviation = $94.9 \mathrm{~mm}$ ). Participants were informed about the aim of the tests and familiarised with the features of the positioning aid prior to the test. After the participants were provided with white tight shorts, socks, bath caps and sports bras for females (Figure 6) they were asked to change into the study clothing. Subsequently, the support elements of the positioning aid were adjusted to their physical dimensions. In line with previous studies (Lu and Wang, 2010; Robinette and Daanen, 2006) three consecutive scans were taken of each subject both with and without the positioning aid in order to determine to what extent the positioning aid reduces postural variability. The subjects were requested to continue breathing normally throughout the scanning process. After each scan, they were asked to relax and then to reposition themselves. The VITUS smart LC 3D body scanner and the anthropometric software Anthroscan used for the development tests were also employed for the study. 


\subsubsection{Reference Test}

A rigid mannequin resembling a human subject was utilised as a reference object. The benefit is that this entirely eliminates the effects of human variability, i.e. respiration, body sway and poor replication of the posture when scanning a human subject. The deviations between multiple scans of a rigid mannequin can be solely ascribed to the precision of the hardware and software of the scanning system if suitably fixed to the floor. Due to the fact that this technical variability is system inherent it constitutes the minimum variability that can be achieved with the experimental set-up and therefore serves as a reference to the effectiveness of the positioning aid. Thus, the study included a reference test comprising of ten repeated scans of a rigid mannequin following the protocol proposed by Kouchi et al. (2012). The mannequin was outfitted with the same attire as the study participants and was placed in the standard standing posture with the feet $35 \mathrm{~cm}$ apart as stipulated by the scanner manufacturer (8).

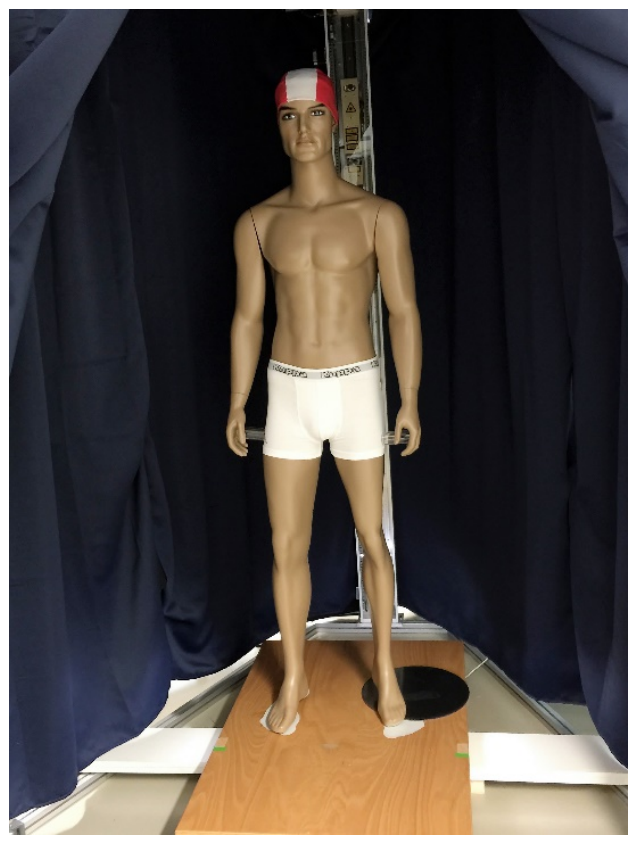

Figure 8: Rigid mannequin (height $1.93 \mathrm{~m}$ ) used for the reference tests

\subsubsection{Rating the effectiveness by gauging sections}

Besides automated extraction of body dimensions from scans the software package "Anthroscan" from Human Solutions allows taking horizontal sections through the scan of the body and measuring distances between any points of the body contour in that section. This software feature allowed the quantification of the variability with and without the positioning aid in the longitudinal and lateral directions. The latter is considered to be essential for the evaluation of the positioning aid and the development of recommendations to further optimise its design. Consequently, the variability with and without positioning aid was determined applying the following methodology: For the first scan out of three the 
standard set of landmarks was extracted from the scans. Extracting the landmarks is an automatic process carried out by the software, which requires no manual interaction.

- Subsequently, scan two and three were superimposed (Figure 9).
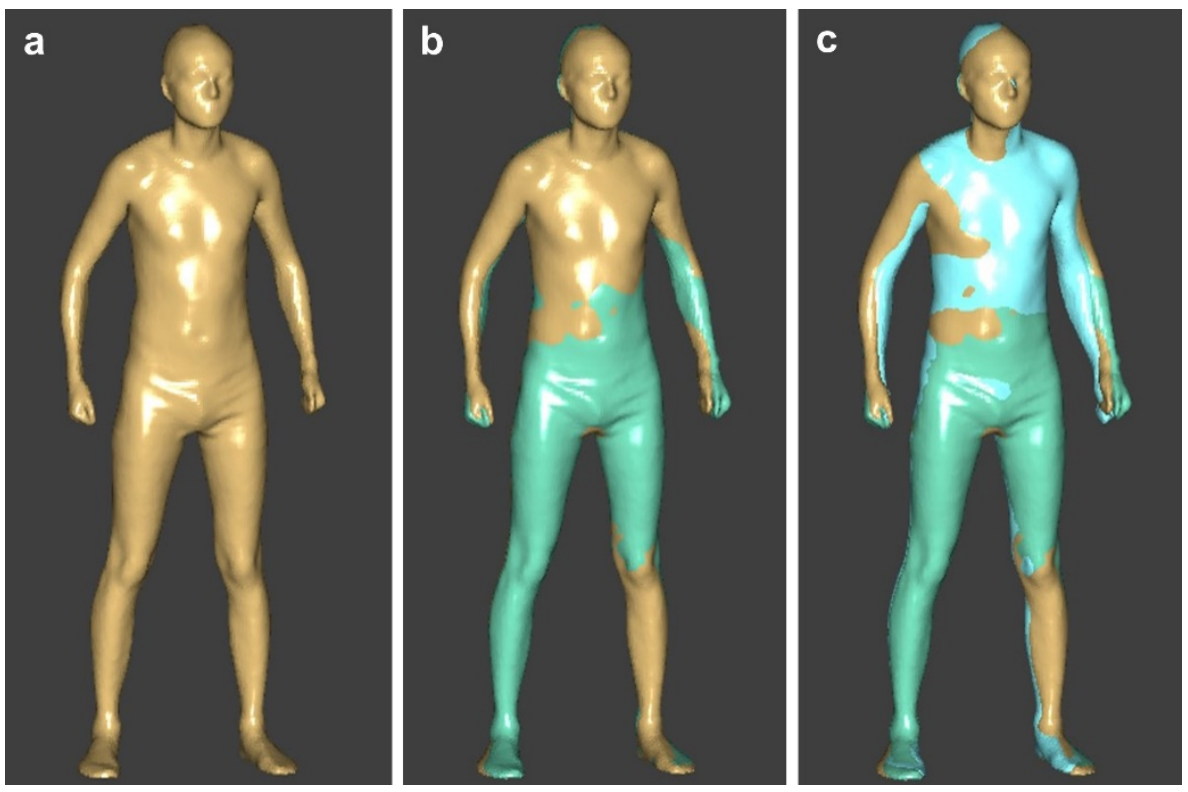

Figure 9: Single scan (a); Scan 1 and 2 superimposed (b); All three scans superimposed (c)

- Horizontal sections were taken through the following seven anatomical landmarks:

- Head front

- Armpit right

- Breast front

- Belly front

- Buttock girth point front

- Thigh crease right

- Minimum leg girth right

The rationale behind the selection of the anatomical landmarks was to ensure that the body parts particularly prone to postural variability (i.e. head, shoulder, arms, legs, hip) are covered to be able to analyse the efficiency of the positioning aid in reducing the variability of these body parts. The sections through the landmarks "breast front" and "belly front" were not just used to quantify the variability of the trunk but also of the upper and the lower arm respectively (Figure 11).

- The maximum lateral distances $(\mathrm{X})$ and longitudinal distances $(\mathrm{Y})$ between the superimposed scans of a section were identified and the distance between the points representing the extremes measured. An example is shown in Figure 10. It compares the sections through the head front landmark of three superimposed scans taken of a subject with (b) and without positioning aid (a). Both, the maximum distances in lateral $(\mathrm{X})$ and in longitudinal direction $(\mathrm{Y})$ between the points of the scans representing the extremes are greater without positioning aid than with positioning aid. 

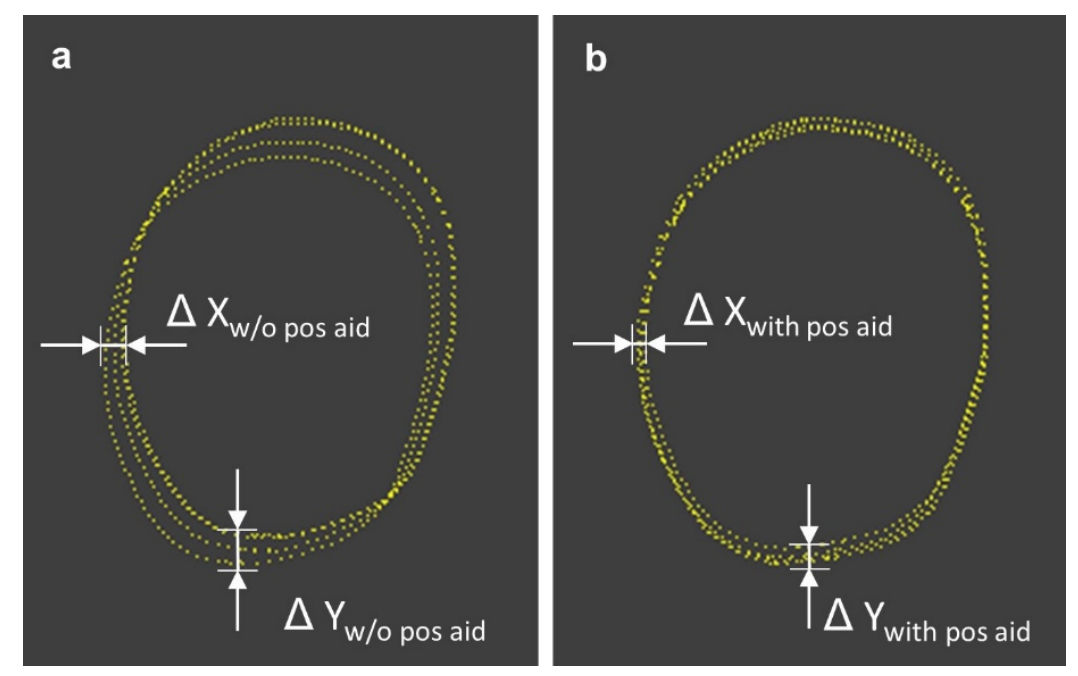

Figure 10: Maximum distances between scans of a section through the "head front" landmark in lateral (X) and longitudinal (Y) direction without positioning aid (a) and with positioning aid (b)

- This approach was applied to the scans of each of the 15 subjects with and without the positioning aid separately in order to compare the average differences in longitudinal and lateral direction in the two experimental conditions.

- Finally, ten scans of the mannequin were taken. The same sections as those used for the human subject scans were reused and the maximum differences between the ten superimposed scans in lateral and longitudinal directions measured to quantify the technical variability.

\subsubsection{Statistical method}

The differences between the mean maximum distances in $\mathrm{X}$ and $\mathrm{Y}$ direction measured with and without positioning aid for the nine selected body parts (18 data sets) were checked for normal distribution using a graphical (histogram) and a quantitative test (Shapiro-Wilk). 16 out of 18 data sets were found to be normally distributed. For these a paired t-test was conducted to evaluate whether the differences across the sample in longitudinal and lateral directions measured with and without the positioning aid were significant. The null hypothesis was that there is no difference between the two experimental conditions and the significance level was set at 0.05 . The two not normally distributed data sets were tested for significance with the Wilcoxon signed rank test based on the same null hypothesis and significance level.

\subsubsection{Analysing the impact on scan extracted measurements}

Besides the rapid and contact free data capture the automatic extraction of body dimensions from the scans is a pivotal asset of state of the art 3D body scanning systems. The Anthroscan software used in this study allows the automatic extraction of 154 body dimensions from scans taken in the standing posture. If the positioning aid had a negative 
impact on the automatic extraction of body dimensions, it would jeopardize this crucial asset and principally put its use in question. Hence, the impact of the positioning aid on the automatic data extraction was examined by comparison of the variability of the body dimensions extracted from the scans captured with the positioning aid with those captured without.

A common approach to analyse the impact on the variability is to compare the mean absolute difference (MAD) of repeated measurements. Thus, the MAD has been utilised in a number of studies as a means to compare the precision of automatic measurements extracted from multiple scans (Dekker, 2000; Lu and Wang, 2010; Robinette and Daanen, 2006).

Consequently, the MAD values of the extracted measurements ascertained with and without positioning aid were calculated to examine whether utilising the positioning aid had an adverse effect on the precision of the automatic measurement extraction.

Furthermore, the MAD values were compared to those published by Dekker (Dekker, 2000) and Lu and Wang (Lu and Wang, 2010) in earlier studies, to show how the precision achieved in this study interrelates with the results of their research in this field.

EN ISO 20685 (2010) as standard for "3-D scanning methodologies for internationally compatible anthropometric databases" lacks recommendations concerning the precision of scan derived measurements. As a consequence, the allowable inter-observer errors for manual measurements established in an error trial for the ANSUR anthropometric survey of the US Army (Gordon et al., 1988) have also become a benchmark for the precision of scan derived measurements (Bradtmiller and Gross, 1999; Lu and Wang, 2010; Robinette and Daanen, 2006). Hence, the MAD values reported in this study were compared with the allowable inter-measurer errors for ANSUR to establish to what extend the results of this study meet the benchmark.

Due to the fact that the sets of anthropometric measurements extracted from the scans in the aforementioned studies differ the 20 measurements showing the greatest commonality were selected for the comparison. The selected measurements included seven ISO 20685 measurements.

\section{Results and Discussion}

\subsection{Effectiveness of the positioning aid}

To evaluate the effectiveness of the positioning aid the mean maximum distances of the sample were calculated and the results produced with and without the positioning aid were compared. Figure 11 shows the extent to which the positioning aid reduced the deviations in lateral $(\mathrm{X})$ and longitudinal direction $(\mathrm{Y})$ between the three superimposed scans for the selected body parts. The paired t-test results indicate that significant differences were found for all body parts in both $\mathrm{X}$ and $\mathrm{Y}$ direction with just one exception (belly front in $\mathrm{X}$ 
direction). The most significant reductions were observed in the variability of the extremities. The variability of the head position (section head front) was reduced by $46 \%$ in lateral and $66 \%$ in longitudinal directions. For the lower arm a reduction of $74 \%(\mathrm{X})$ and $63 \%(\mathrm{Y})$ and for the leg (section minimum leg girth) of $57 \%(\mathrm{X})$ and $63 \%(\mathrm{Y})$ was achieved. It is apparent from the data presented that none of the differences between the scans is greater than $9 \mathrm{~mm}$ in the lateral direction and $11 \mathrm{~mm}$ in longitudinal direction when using the positioning aid. It can be observed that the variability when using the positioning aid is between two and three times higher than the resolution of the body scanner (2-4 mm) for all body parts.

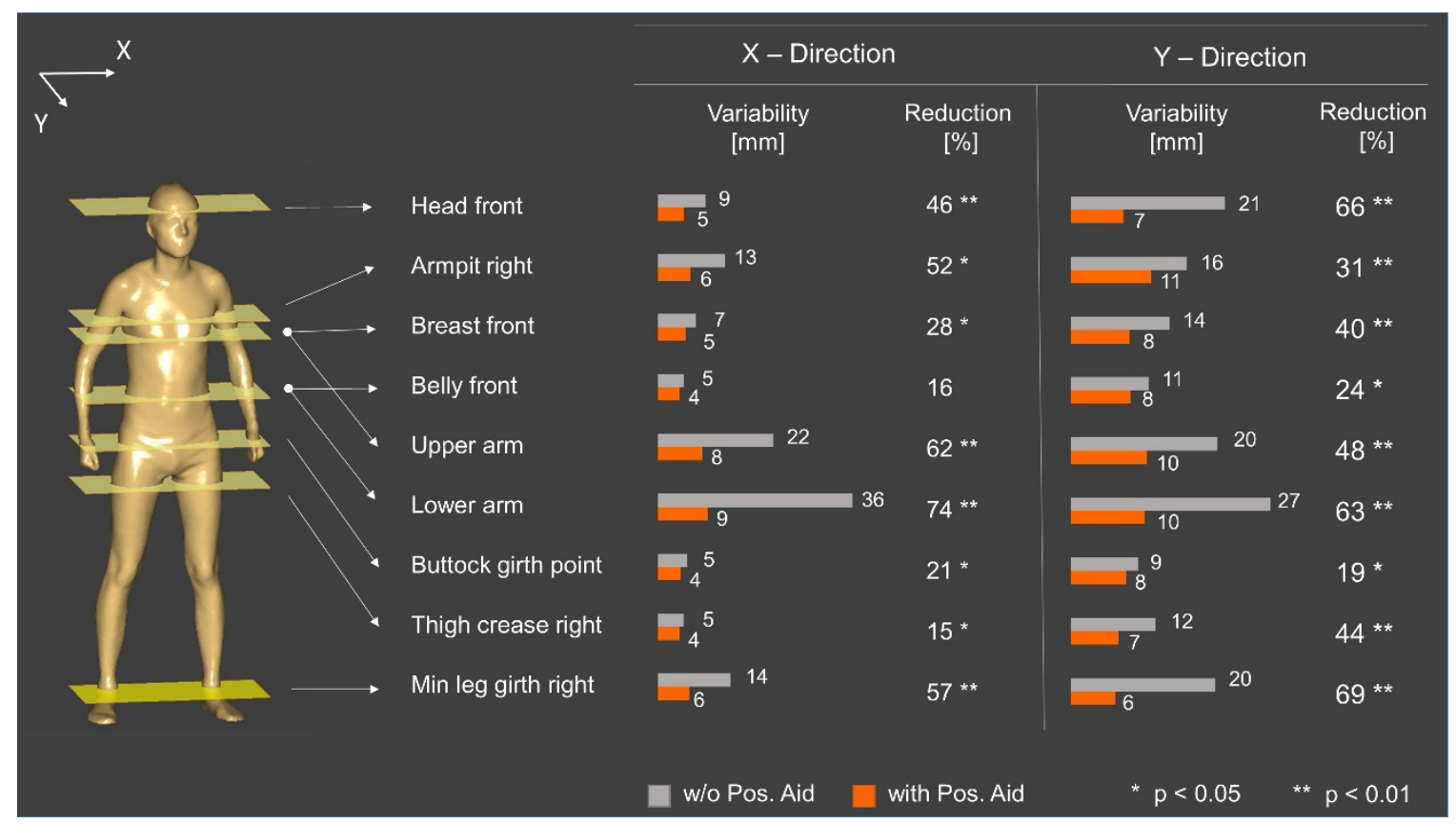

Figure 11: Average variability of the sample with and w/o positioning aid

\subsection{Contribution of the technical variability to the overall variability}

The residual variability when using the positioning aid encompasses the remaining human variability (body sway, respiration and postural variations) as well as the technical variability (software and hardware). Figure12 shows the results of the reference test conducted with a rigid mannequin to quantify to what extent the technical variability contributes to the residual variability. It becomes apparent that up to $5 \mathrm{~mm}$ of the residual variability in both, lateral as well as longitudinal direction can be ascribed to the precision of the hardware and the performance of the scanning software. In other words, the system inherent technical variability contributes between $25 \%$ and $100 \%$ to the residual variability in the lateral and between $29 \%$ and $67 \%$ in the longitudinal directions. The share of the technical variability in the residual variability was least for the upper and lower arms in both directions and for the armpit in the longitudinal direction. 


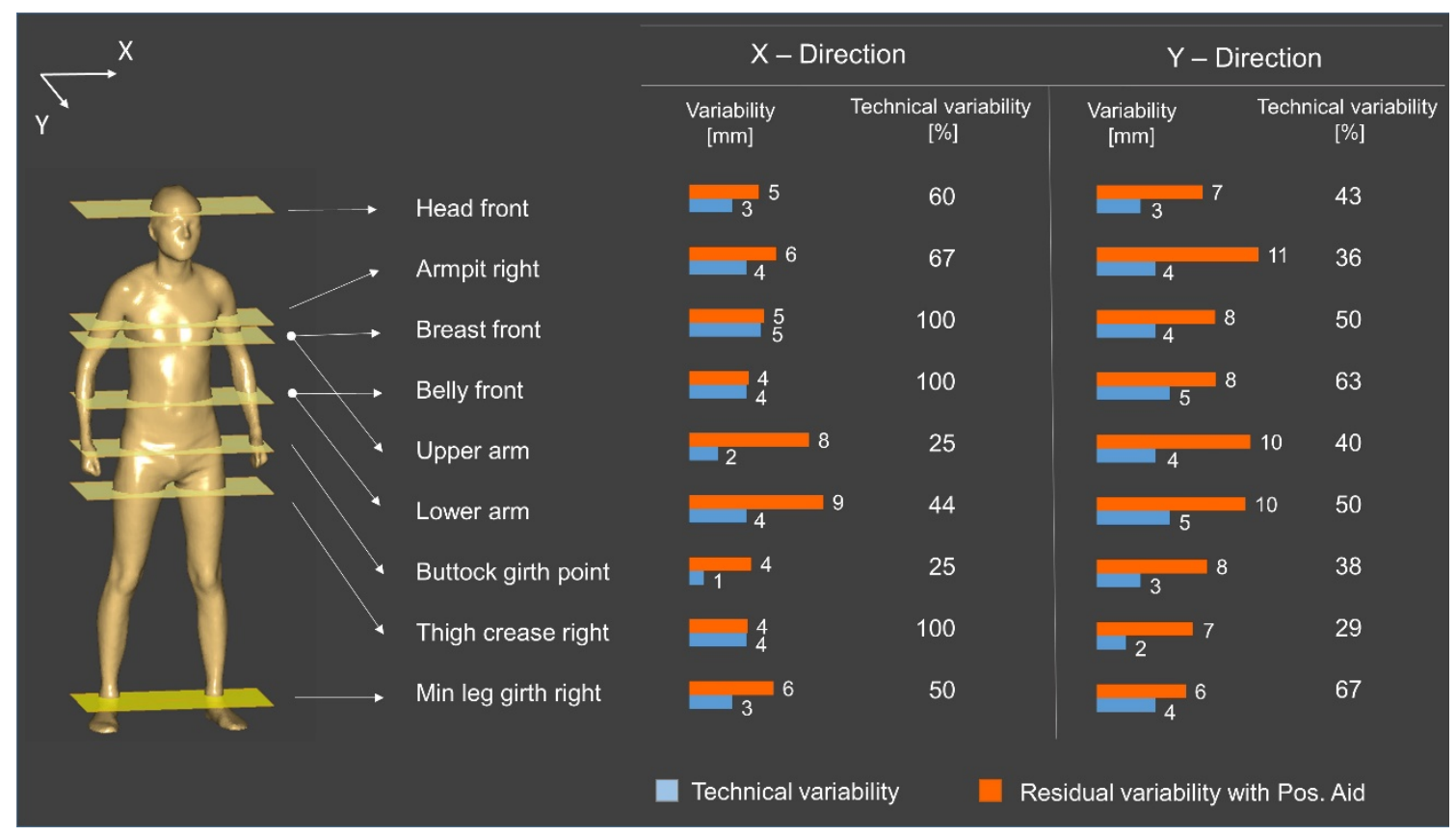

Figure12: Contribution of technical variability to residual variability with positioning aid

\subsection{Impact on scan derived measurements}

Table 1 presents the MAD values of the sample for twenty selected body dimensions from three repeated scans ascertained with and without the positioning aid. Furthermore, it compares these MAD values with those reported by Dekker (2000) and Lu \& Wang (2010) as well as with the ANSUR allowable inter-observer error (Gordon et al., 1988).

It is apparent from this table that for twelve of the body dimensions the MAD values with positioning aid were lower than without suggesting that the positioning aid increased the precision for $66.7 \%$ of the selected measurements. This result is roughly in line with the ratio obtained for the complete set of 154 automatically extracted body dimensions by the scanner software. For 110 out of the 154 body dimensions the variability decreased (71.4\%). Table 1 indicates that the biggest reduction of variability was observed for the bust and under bust girths. The differences observed for body dimensions representing small circumferences and distances were generally smaller. In the majority of cases the differences between the MAD values ascertained with and without positioning aid were smaller than $2 \mathrm{~mm}$. The comparison with the MAD values observed by Dekker and Lu \& Wang (Dekker, 2000; Lu and Wang, 2010) suggests that the precision of this study lies well in-between the precision achieved in their studies.

Table 1 includes the ANSUR allowable inter-observer error for 14 of the scan-extracted body dimensions constituting the benchmark for the precision of repeated measurements. The comparison with the MAD values of this study reveals that utilising the positioning aid only one measurement (right thigh girth) exceeded the allowable ANSUR inter-observer error by less than $1 \mathrm{~mm}$. Without positioning aid three MAD values exceeded the allowable ANSUR benchmark. 
Table I: Comparison of Mean Absolute Differences (MAD)

\begin{tabular}{|c|c|c|c|c|c|c|}
\hline \multirow{2}{*}{ Body dimension } & \multicolumn{2}{|c|}{$\begin{array}{l}\text { MAD } \\
(\mathrm{mm})\end{array}$} & \multirow{2}{*}{$\begin{array}{c}\Delta \\
\text { MAD with } \\
- \\
\text { MAD w/o } \\
(\mathrm{mm})\end{array}$} & \multirow{2}{*}{$\begin{array}{l}\text { MAD } \\
\text { (mm) } \\
\text { Dekker }\end{array}$} & \multirow{2}{*}{$\begin{array}{l}\text { MAD } \\
(\mathrm{mm}) \\
\text { Lu \& } \\
\text { Wang }\end{array}$} & \multirow{2}{*}{$\begin{array}{c}\text { ANSUR } \\
\text { allowable } \\
\text { inter- } \\
\text { observer } \\
\text { error (mm) }\end{array}$} \\
\hline & $\begin{array}{c}\text { w/o } \\
\text { pos aid }\end{array}$ & $\begin{array}{c}\text { with } \\
\text { pos aid }\end{array}$ & & & & \\
\hline Body height ${ }^{1}$ & 2.22 & 2.89 & 0.67 & 4.00 & 2.08 & 7.00 \\
\hline Crotch height ${ }^{1}$ & 6.62 & 7.69 & 1.07 & 4.00 & & 10.00 \\
\hline Waist height & $4.31 *$ & 3.87 & -0.44 & 6.00 & & 4.00 \\
\hline Knee height & 2.76 & 1.78 & -0.98 & 4.00 & & 6.00 \\
\hline Hip height & 11.70 & 12.80 & 1.10 & 3.00 & & \\
\hline Mid neck girth ${ }^{1}$ & 4.00 & 3.64 & -0.36 & 6.00 & & 6.00 \\
\hline Bust/chest girth ${ }^{1}$ & 10.49 & 6.44 & -4.05 & 6.00 & 6.03 & 15.00 \\
\hline Under bust circumference & $16.93^{*}$ & 11.38 & -5.55 & 7.00 & & 16.00 \\
\hline Waist girth ${ }^{1}$ & 5.51 & 9.87 & 4.36 & 9.00 & 5.13 & 11.00 \\
\hline Higher hip girth & 6.80 & 6.36 & -0.44 & 11.00 & & \\
\hline Hip girth & 3.87 & 4.44 & 0.57 & 2.00 & 5.47 & \\
\hline Right thigh girth ${ }^{1}$ & 5.47 & $6.76^{*}$ & 1.29 & 3.00 & & 6.00 \\
\hline Right knee girth & 3.60 & 2.89 & -0.71 & 3.00 & & 4.00 \\
\hline Right calf girth ${ }^{1}$ & 1.38 & 1.07 & -0.31 & 2.00 & & 5.00 \\
\hline Right min. leg girth & 5.60 & 2.71 & -2.89 & 2.00 & & \\
\hline Bust point width & 4.00 & 2.86 & -1.14 & 15.00 & & 10.00 \\
\hline Across back width & 4.98 & 5.78 & 0.80 & 21.00 & 6.71 & 13.00 \\
\hline Neck to waist center back & 4.04 & 4.44 & 0.40 & 17.00 & 3.53 & 5.00 \\
\hline Neck to across back width & $7.42 *$ & 3.91 & -3.51 & 11.00 & & 4.00 \\
\hline Crotch length & 13.69 & 12.22 & -1.47 & 16.00 & & 16.00 \\
\hline Mean & 6.27 & 5.69 & -0.58 & 7.60 & 4.83 & 8.63 \\
\hline
\end{tabular}

${ }^{1}$ EN ISO 20685 recommended measurement

* Value exceeds allowable ANSUR inter-observer error

\subsection{Discussion}

The results of the evaluation of seven horizontal sections suggest that the positioning aid efficiently reduced the maximum distances between multiple scans for all body parts in both lateral as well as longitudinal directions. The positioning aid was shown to be particularly efficient in reducing the variability of head, arms and legs. It therefore can be assumed that the fixation elements selected for the positioning aid help subjects to reliably replicate the standard scanning posture when scanned repeatedly and also efficiently reduce body sway during the scanning process.

The residual variability measured with the positioning aid encompasses the remaining human variability as well as the technical variability. The reference test with a rigid mannequin showed that the technical variability has a considerable share in the residual variability. However, its contribution to the residual variability differs for different body parts. The greatest difference between technical and residual variability was measured for 
the armpit, upper arm and lower arm section, indicating that there is an opportunity for further improvement of the positioning aid for these body parts. Further analysis of the armpit sections (Figure 13) revealed that the comparably high residual variability was caused by "twisted shoulders". Differences in longitudinal direction of more than $10 \mathrm{~mm}$ between the right and the left arm indicated that 4 of the 15 subjects had difficulties in reliably repositioning their shoulder correctly in the lateral plane.

This twisting of the shoulders considerably increases the deviation in the longitudinal direction for the armpit section but also has an adverse impact on the precise repositioning of trunk and arms. Hence, the introduction of an additional fixation element for the shoulder needs to be seriously considered. An option for further reducing the variability of the upper and lower arm is to adjust the handles of the positioning aid at a height, which ensures that the arms are stretched. This measure potentially reduces deviations due to varying elbow abduction.

The fact that the residual variability for the breast front and the belly front section ranked among the lowest compared to other body parts indicates that respiration did not noticeably contribute to the residual variability. It therefore can be concluded that asking the subjects to continue breathing quietly as stipulated in ISO 20685 led to the desired effect.
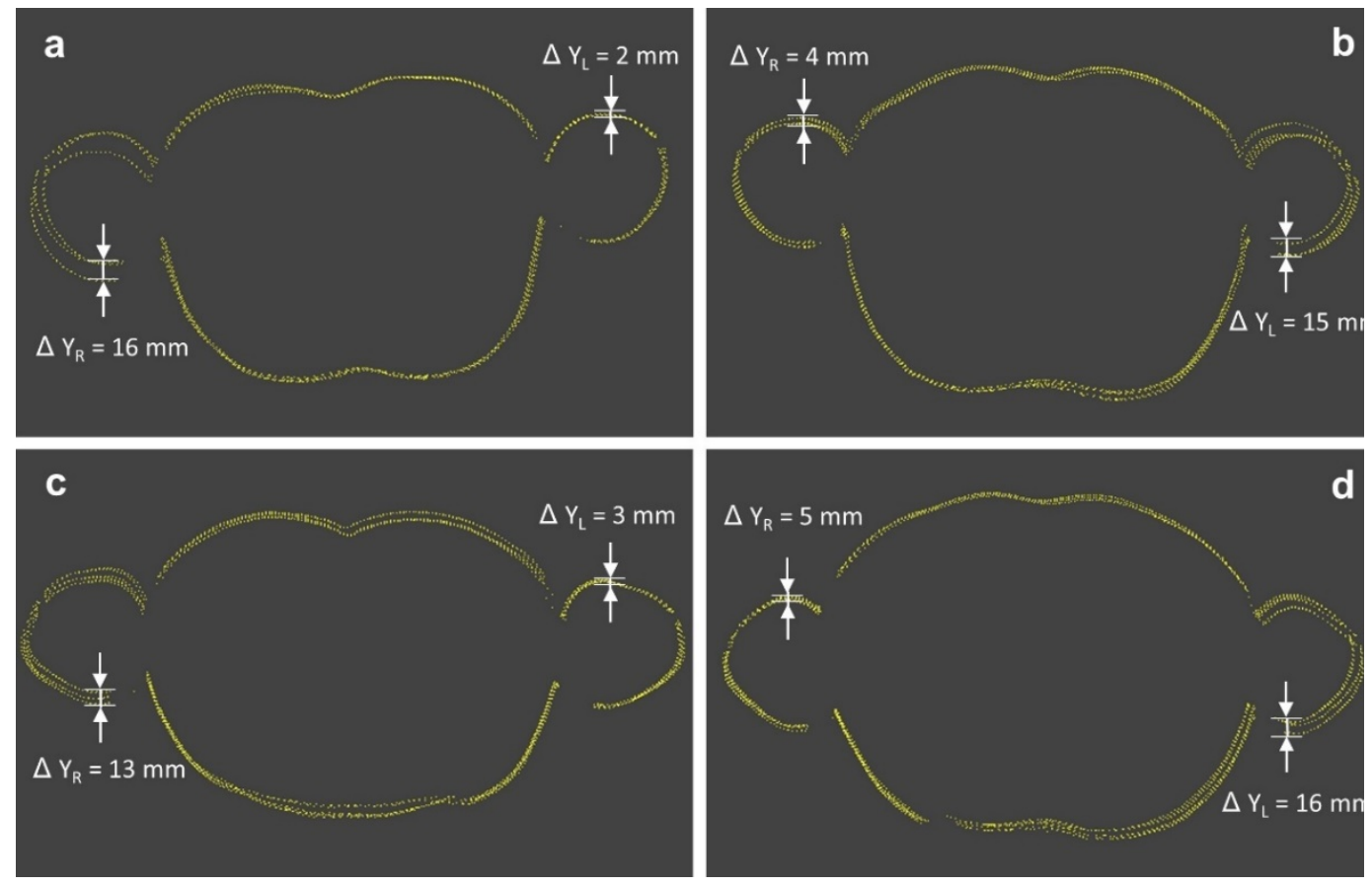

Figure 13: Sections through the armpit landmark with differences in the $Y$-axis of more than $10 \mathrm{~mm}$ between the right and the left arm indicated poor repositioning of the shoulder in four cases (a-d)

The evaluation of the MAD values of 20 automatically extracted measurements suggests that the positioning aid did not prevent the software-assisted measurement extraction but overall even increased the precision of the results. The precision observed in this study lies between the upper and lower limits of the precision range established by Dekker and Lu \& Wang in earlier studies. However, the significance of the comparison with the results of $\mathrm{Lu}$ 
\& Wang is limited since only six of the measurements taken in their study are identical with the 20 selected measurements considered in this study. The comparison with the ANSUR allowable inter-observer error as benchmark for the precision of repeated scan extracted measurements underlined the positive effect of the positioning aid. Whilst without positioning aid three out of 20 measured body dimensions exceeded the ANSUR benchmark employing the positioning aid reduced the rate to just one minor exceedance.

\section{Conclusions and further work}

In this paper the development of a positioning aid is described that effectively reduces postural variability for 3D body scanning systems and at the same time does not compromise scan quality, data integrity and the automatic extraction from scans.

The evaluation of the effectiveness of the positioning aid by evaluating the consistency shown in seven horizontal sections taken through the body of the scans revealed that it significantly reduces the human variability for all body parts in both, lateral and longitudinal directions. The largest reductions were found in the variability of the extremities, i.e., head, arms and legs.

A reference test with a rigid mannequin was conducted to entirely eliminate the human variability in order to quantify the contribution of the technical variability to the residual variability when utilising the positioning aid. It was shown that the technical variability resulting from the hardware precision and the performance of the scanning software contributes significantly to the residual variability. This implies that the potential for the positioning aid to further reduce human variability is limited. However, a detailed analysis of the sections taken through the armpit landmark revealed that the variability of shoulders and arms can be further reduced by incorporating a supporting element, which reliably avoids subjects twisting their shoulders.

The assessment of the MAD calculated from automatically extracted measurements has shown that the positioning aid had no adverse effect on the automatic measurement extraction and even improved the precision of its results. The comparison with the ANSUR allowable inter-observer error suggests that utilising the positioning aid helps meeting this benchmark for repeated scan-derived measurements.

The evaluation of the accuracy, i.e. the extent to which measurements extracted from 3D scans approximate the appropriate manual measurements, has already been subject of numerous studies (Han et al., 2010; Kuehnapfel et al., 2016; Lu and Wang, 2010). However, holding the handles of the arm fixture might lead to a slightly different body posture resulting in deviations from the findings ascertained with subjects freely standing in the scanner. Further work is required to clarify a possible impact.

Recently introduced scanners such as the "VITUS bodyscan" (VITRONIC), the "3D Scamera" (OSENSUS) or the "3dMDbody System" (3dMD) provide high-resolution scans at reduced scanning times. Due to the reduced scanning times body sway becomes negligible and does not contribute to human variability anymore. Furthermore, the high resolution might lead to artefacts when using the positioning aid, which require a further 
design iteration. It is therefore recommended to explore potential implications in future studies.

Overall, this study strengthens the idea that the newly developed positioning aid reduces the postural variability to an extent that allows the precise superimposition of scans taken from the same subject at different occasions or in diverse configurations. Hence, the logical progression of the research described in this paper would be to test the suitability of the positioning aid for e.g. medical applications or clothed anthropometry.

\section{Funding}

This research is the result of self-funded $\mathrm{PhD}$ research and did not receive any specific grant from funding agencies in the public, commercial, or not-for-profit sectors.

\section{References}

Bradtmiller, B., Gross, M., 1999. 3D whole-body scans: Measurement extraction software validation. SAE Trans. doi:10.4271/1999-01-1892

D’Apuzzo, N., 2007. 3D body scanning technology for fashion and apparel industry, in: Beraldin, J.-A., Remondino, F., Shortis, M.R. (Eds.), SPIE. p. 64910O-64910O-12. doi:10.1117/12.703785

Daanen, H.A.M., Brunsman, M. a., Robinette, K.M., 1997. Reducing movement artifacts in whole body scanning, in: Proceedings. International Conference on Recent Advances in 3-D Digital Imaging and Modeling (Cat. No.97TB100134). IEEE Comput. Soc. Press, pp. 262-265. doi:10.1109/IM.1997.603874

Daanen, H.A.M., van de Water, G.J., 1998. Whole body scanners. Displays 19, 111-120. doi:10.1016/S0141-9382(98)00034-1

Dekker, L., 2000. 3D Human Body Modelling from Range Data. University College London.

EN ISO, 2010. 20685: 3-D scanning methodologies for internationally compatible anthropometric databases. Geneva Int. Stand. Organ.

Gordon, C.C., Churchill, T., Clauser, C.E., Mcconville, J.T., Tebbetts, I., Walker, R.A., 1988. 1988 Anthropometric Survey of U. S . Army Personnel : Methods and Summary Statistics. Security 640.

Guitierrez, C. M., Gallagher, P.D., 2008. Pilot Study of Firefighter Three- Dimensional Anthropometry to Improve Seatbelt Safety (Report NIST 08-919). Gaithersburg.

Han, H., Nam, Y., Choi, K., 2010. Comparative analysis of 3D body scan measurements and manual measurements of size Korea adult females. Int. J. Ind. Ergon. 40, 530-540. doi:10.1016/j.ergon.2010.06.002

Hsiao, H., Whitestone, J., Kau, T.-Y., Whisler, R., Routley, J.G., Wilbur, M., 2014. Sizing firefighters: method and implications. Hum. Factors 56, 873-910.

Jones, M.L.H., Kim, K.H., Keefe, A.A., Farrell, P.S.E., Bossi, L.M., 2015. Proceedings 19 1-7.

Kouchi, M., Mochimaru, M., 2011. Errors in landmarking and the evaluation of the accuracy of traditional and 3D anthropometry. Appl. Ergon. 42, 518-27. doi:10.1016/j.apergo.2010.09.011

Kouchi, M., Mochimaru, M., 2008. Evaluation of accuracy in traditional and 3D anthropometry, 
in: SAE Technical Paper Series. SAE International. doi:10.4271/2008-01-1882

Kouchi, M., Mochimaru, M., Bradtmiller, B., Daanen, H.A.M., Li, P., Nacher, B., Nam, Y., 2012. A protocol for evaluating the accuracy of 3D body scanners. Work 41 Suppl 1, 4010-7. doi:10.3233/WOR-2012-0064-4010

Kuehnapfel, A., Ahnert, P., Loeffler, M., Broda, A., Scholz, M., 2016. Reliability of 3D laser-based anthropometry and comparison with classical anthropometry. Sci. Rep. 6, 26672. doi:10.1038/srep26672

Lu, J.-M., Wang, M.-J.J., 2010. The Evaluation of Scan-Derived Anthropometric Measurements. IEEE Trans. Instrum. Meas. 59, 2048-2054. doi:10.1109/TIM.2009.2031847

Lu, J.-M., Wang, M.-J.J., Mollard, R., 2010. The effect of arm posture on the scan-derived measurements. Appl. Ergon. 41, 236-41. doi:10.1016/j.apergo.2009.07.002

Mckinnon, L., Istook, C.L., 2002. Body scanning: The effects of subject respiration and foot positioning on the data integrity of scanned measurements. J. Fash. Mark. Manag. 6, 103121. doi:10.1108/13612020210429458

Reed, M.P., 2012. A Pilot Study of Three-Dimensional Child Anthropometry for Vehicle Safety Analysis. Proc. Hum. Factors Ergon. Soc. Annu. Meet. 56, 2326-2330. doi:10.1177/1071181312561489

Robinette, K., Daanen, H.A.M., 2006. Precision of the CAESAR scan-extracted measurements. Appl. Ergon. 37, 259-65. doi:10.1016/j.apergo.2005.07.009

Stewart, A., Ledingham, R., Furnace, G., Schranz, N., Nevill, A., 2016. The ability of UK offshore workers of different body size and shape to egress through a restricted window space. Appl. Ergon. 55, 226-233. doi:10.1016/j.apergo.2015.11.005

Tomkinson, G.R., Shaw, L.G., 2013. Quantification of the postural and technical errors in asymptomatic adults using direct 3D whole body scan measurements of standing posture. Gait Posture 37, 172-177. doi:10.1016/j.gaitpost.2012.06.031

Treleaven, P., 2004. Sizing us up. IEEE Spectr. 41, 29-31. doi:10.1109/MSPEC.2004.1279190 